\title{
A cut-off in the TeV gamma-ray spectrum of the SNR Cassiopeia A
}

MAGIC Collaboration: M. L. Ahnen, ${ }^{1}$ S. Ansoldi, ${ }^{2,21}$ L. A. Antonelli, ${ }^{3}$ C. Arcaro, ${ }^{4}$ A. Babić, ${ }^{5}$ B. Banerjee, ${ }^{6}$ P. Bangale,${ }^{7}$ U. Barres de Almeida, ${ }^{7}$ J. A. Barrio, ${ }^{8}$

J. Becerra González, ${ }^{9}$ W. Bednarek,${ }^{10}$ E. Bernardini, ${ }^{11,12}$ A. Berti, ${ }^{2}$ W. Bhattacharyya,${ }^{11}$ B. Biasuzzi, ${ }^{2}$ A. Biland, ${ }^{1}$ O. Blanch,${ }^{13}$ S. Bonnefoy, ${ }^{8}$ G. Bonnoli, ${ }^{14}$ R. Carosi,${ }^{14}$ A. Carosi, ${ }^{3}$ A. Chatterjee, ${ }^{6}$ M. Colak, ${ }^{13}$ P. Colin, ${ }^{7}$ E. Colombo, ${ }^{9}$ J. L. Contreras, ${ }^{8}$ J. Cortina, ${ }^{13}$ S. Covino, ${ }^{3}$ P. Cumani, ${ }^{13}$ P. Da Vela,${ }^{14}$ F. Dazzi,${ }^{3}$ A. De Angelis, ${ }^{4}$ B. De Lotto ${ }^{2}$ E. de Oña Wilhelmi, ${ }^{15 \star}$ F. Di Pierro,${ }^{4}$ M. Doert, ${ }^{16}$ A. Domínguez, ${ }^{8}$ D. Dominis Prester, ${ }^{5}$ D. Dorner, ${ }^{17}$ M. Doro, ${ }^{4}$ S. Einecke, ${ }^{16}$ D. Eisenacher Glawion, ${ }^{17}$ D. Elsaesser, ${ }^{16}$ M. Engelkemeier, ${ }^{16}$ V. Fallah Ramazani, ${ }^{18}$ A. Fernández-Barral, ${ }^{13}$ D. Fidalgo, ${ }^{8}$ M. V. Fonseca, ${ }^{8}$ L. Font, ${ }^{19}$ C. Fruck, ${ }^{7}$ D. Galindo,${ }^{20 \star}$ R. J. García López, ${ }^{9}$ M. Garczarczyk, ${ }^{11}$ M. Gaug, ${ }^{19}$ P. Giammaria, ${ }^{3}$ N. Godinović,${ }^{5}$ D. Gora,,${ }^{11}$ D. Guberman, ${ }^{13 \star}$ D. Hadasch, ${ }^{21}$ A. Hahn, ${ }^{7}$ T. Hassan, ${ }^{13}$ M. Hayashida, ${ }^{21}$ J. Herrera, ${ }^{9}$ J. Hose ${ }^{7}$ D. Hrupec,${ }^{5}$ T. Inada,${ }^{21}$ K. Ishio, ${ }^{7}$ Y. Konno, ${ }^{21}$ H. Kubo,${ }^{21}$ J. Kushida, ${ }^{21}$ D. Kuveždić, ${ }^{5}$ D. Lelas,${ }^{5}$ E. Lindfors, ${ }^{18}$ S. Lombardi, ${ }^{3}$ F. Longo, ${ }^{2}$ M. López, ${ }^{8}$ C. Maggio, ${ }^{19}$ P. Majumdar, ${ }^{6}$ M. Makariev, ${ }^{22}$ G. Maneva, ${ }^{22}$ M. Manganaro, ${ }^{9}$ K. Mannheim, ${ }^{17}$ L. Maraschi, ${ }^{3}$ M. Mariotti, ${ }^{4}$ M. Martínez, ${ }^{13}$ D. Mazin,,${ }^{7,21}$ U. Menzel, ${ }^{7}$ M. Minev, ${ }^{22}$ R. Mirzoyan, ${ }^{7}$ A. Moralejo, ${ }^{13}$ V. Moreno, ${ }^{19}$ E. Moretti, ${ }^{7}$ V. Neustroev, ${ }^{18}$ A. Niedzwiecki, ${ }^{10}$ M. Nievas Rosillo, ${ }^{8}$ K. Nilsson, ${ }^{18}$ D. Ninci, ${ }^{13}$ K. Nishijima, ${ }^{21}$ K. Noda, ${ }^{13}$ L. Nogués, ${ }^{13}$ S. Paiano,${ }^{4}$ J. Palacio, ${ }^{13}$ D. Paneque,${ }^{7}$ R. Paoletti, ${ }^{14}$ J. M. Paredes, ${ }^{20}$ G. Pedaletti, ${ }^{11}$ M. Peresano, ${ }^{2}$ L. Perri, ${ }^{3}$ M. Persic, $, 2,3$

P. G. Prada Moroni ${ }^{23}$ E. Prandini, ${ }^{4}$ I. Puljak, ${ }^{5}$ J. R. Garcia,${ }^{7}$ I. Reichardt, ${ }^{4}$ W. Rhode, ${ }^{16}$ M. Ribó, ${ }^{20}$ J. Rico, ${ }^{13}$ C. Righi, ${ }^{3}$ T. Saito, ${ }^{21}$ K. Satalecka, ${ }^{11}$ S. Schroeder, ${ }^{16}$ T. Schweizer, ${ }^{7}$ S. N. Shore,$^{23}$ J. Sitarek, ${ }^{10}$ I. Šnidarić, ${ }^{5}$ D. Sobczynska, ${ }^{10}$ A. Stamerra, ${ }^{3}$ M. Strzys, ${ }^{7}$ T. Surić, ${ }^{5}$ L. Takalo, ${ }^{18}$ F. Tavecchio, ${ }^{3}$ P. Temnikov, ${ }^{22}$ T. Terzić,${ }^{5}$ D. Tescaro, ${ }^{4}$ M. Teshima, ${ }^{7,21}$ N. Torres-Albà, ${ }^{20}$ A. Treves, ${ }^{2}$ G. Vanzo, ${ }^{9}$ M. Vazquez Acosta, ${ }^{9}$ I. Vovk, ${ }^{7}$ J. E. Ward, ${ }^{13}$ M. Will ${ }^{7}$ and D. Zarić ${ }^{5}$

Affiliations are listed at the end of the paper

Accepted 2017 August 8. Received 2017 August 8; in original form 2017 July 3

\section{ABSTRACT}

It is widely believed that the bulk of the Galactic cosmic rays is accelerated in supernova remnants (SNRs). However, no observational evidence of the presence of particles of $\mathrm{PeV}$ energies in SNRs has yet been found. The young historical SNR Cassiopeia A (Cas A) appears as one of the best candidates to study acceleration processes. Between 2014 December and 
2016 October, we observed Cas A with the MAGIC telescopes, accumulating $158 \mathrm{~h}$ of good quality data. We derived the spectrum of the source from $100 \mathrm{GeV}$ to $10 \mathrm{TeV}$. We also analysed $\sim 8 \mathrm{yr}$ of Fermi-LAT to obtain the spectral shape between $60 \mathrm{MeV}$ and $500 \mathrm{GeV}$. The spectra measured by the LAT and MAGIC telescopes are compatible within the errors and show a clear turn-off $(4.6 \sigma)$ at the highest energies, which can be described with an exponential cut-off at $E_{c}=3.5\left({ }_{-1.0}^{+1.6}\right)_{\text {stat }}\left({ }_{-0.9}^{+0.8}\right)_{\text {sys }} \mathrm{TeV}$. The gamma-ray emission from $60 \mathrm{MeV}$ to $10 \mathrm{TeV}$ can be attributed to a population of high-energy protons with a spectral index of $\sim 2.2$ and an energy cut-off at $\sim 10 \mathrm{TeV}$. This result indicates that Cas $\mathrm{A}$ is not contributing to the high energy $(\sim \mathrm{PeV})$ cosmic ray sea in a significant manner at the present moment. A one-zone leptonic model fails to reproduce by itself the multiwavelength spectral energy distribution. Besides, if a non-negligible fraction of the flux seen by MAGIC is produced by leptons, the radiation should be emitted in a region with a low magnetic field $(B \lesssim 180 \mu \mathrm{G})$ like in the reverse shock.

Key words: acceleration of particles - cosmic rays - ISM: supernova remnants - gamma rays: general.

\section{INTRODUCTION}

Supernova remnants (SNRs) are widely believed to be able to accelerate cosmic rays (CRs) to PeV energies, and of being the main contributors to the galactic CR sea (Berezhko, Pühlhofer \& Völk 2003; Bell et al. 2013; O'C. Drury 2014). Two arguments support this belief. On one hand, SNRs can explain the observed energy density of CRs if one assumes that around 10 percent of the kinetic energy of the supernova ( $\mathrm{SN}$ ) explosion goes into $\mathrm{CR}$ acceleration and a supernova explosion rate of $\sim 3$ per century (Ginzburg 1964; Gaisser 1991). On the other hand, Diffusive Shock Acceleration (DSA, Axford, Leer \& Skadron 1977; Krymskii 1977; Bell 1978a,b; Blandford \& Ostriker 1978; Bell 2013) offers a plausible acceleration mechanism and explains the CR spectral shape. Observations at high and very high energies have further strengthened this paradigm: several SNRs have been observed to emit at TeV energies, a signature that particles are being accelerated to relativistic energies. The spectral shape observed in some SNRs at sub-GeV energies points to neutral pion decay as the origin of the high-energy emission (Ackermann et al. 2013; Aharonian 2013); however, the origin of the emission at the highest energies, in the $\mathrm{TeV}$ regime, is still uncertain.

If SNRs are the sources of all galactic CRs, they must be able to accelerate particles all the way up to the knee of the CR spectrum, a feature observed at around $3 \mathrm{PeV}$. In fact, this represented an important theoretical challenge for decades because standard DSA was unable to explain acceleration beyond $100 \mathrm{TeV}$. It has been later realized (Bell 2004) that the magnetic field upstream of the shock of young SNR can be amplified due to instabilities produced by CRs themselves. The missing part to solve the paradox is the observational evidence: as of today, no SNR has been found where hadronic $\mathrm{CR}$ acceleration up to $\mathrm{PeV}$ energies can be firmly established.

Cassiopeia A (Cas A) is one of the few good candidates for these studies. The precise knowledge of the age of this core-collapse SNR (330 yr), the remnant of a historical supernova in AD 1680, allows the determination of many otherwise free parameters when studying its morphology and spectral shape. It is located at a distance of $3.4_{-0.1}^{+0.3} \mathrm{kpc}$ and has an angular diameter of 5 arcmin (Reed et al. 1995). It is the brightest radio source outside our Solar system and it is in fact bright all over the electromagnetic spectrum, offering an excellent opportunity to study particle acceleration.

Cas A has been extensively observed in radio wavelengths (Lastochkin et al. 1963; Medd \& Ramana 1965; Allen \& Barrett 1967; Parker 1968; Braude et al. 1969; Hales et al. 1995).
Most of the emission comes from a bright radio ring of $\sim 1.7 \mathrm{pc}$ radius and a faint outer plateau of $\sim 2.5 \mathrm{pc}$ radius (Zirakashvili et al. 2014), although a distinct emission coming from several compact and bright knots has also been identified (Anderson et al. 1991). The spectral index of the radio flux can vary from $\sim 0.6$ to $\sim 0.9$ over the remnant. Several emission regions were also identified in the X-ray band (Gotthelf et al. 2001; Maeda et al. 2009; Grefenstette et al. 2015; Wang \& Li 2016). In the gamma-ray domain, FermiLAT detected the source at GeV energies (Abdo et al. 2010) and later derived a spectrum that displays a low energy spectral break at $1.72 \pm 1.35 \mathrm{GeV}$ (Yuan et al. 2013). In the TeV energy range, Cas A was first detected by HEGRA (Aharonian et al. 2001) and later confirmed by MAGIC (Albert et al. 2007). VERITAS has recently reported a spectrum extending well above $1 \mathrm{TeV}(\mathrm{Ku}-$ mar 2015; Holder et al. 2017), where the measured spectral index is larger than the Fermi-LAT index of $2.17 \pm 0.09$. The spectrum seems to steepen from the Fermi-LAT energy range to the TeV bands, according to all IACT measurements. Still, the statistical and systematic errors are too large for a final conclusion.

Multiwavelength modelling of Cas A observations has not yet resulted in a clear discrimination between hadronic and/or leptonic origin of the observed radiation in the $\mathrm{GeV}$ to $\mathrm{TeV}$ energy range (i.e. Berezhko et al. 2003; Vink \& Laming 2003; Yuan et al. 2013; Saha et al. 2014; Zirakashvili et al. 2014). However, the break in the Fermi-LAT spectrum at $\sim 1 \mathrm{GeV}$ combined with the observations at $\mathrm{TeV}$ energies suggests that the observed gamma-ray flux has either a pure hadronic origin or that several emission mechanisms (proton-proton interaction, inverse Compton and/or bremsstrahlung) are involved. Indeed, several plausible acceleration regions have been identified in Cas A. Chandra X-ray images (Gotthelf et al. 2001) and high-resolution Very Large Array (VLA) radio synchrotron maps (Anderson \& Rudnick 1995) show a thin outer edge to the SNR that has been interpreted to represent the forward shock where the blast wave encounters the circumstellar medium (DeLaney \& Rudnick 2003). The cold SNR ejecta expands supersonically outward from the explosion centre producing a strong shock where its relatively high magnetic field (Cowsik \& Sarkar 1980) can be amplified and hence accelerate CRs to PeV energies (Bell 2004, 2013). This scenario was reinforced by the observations of year-scale variability in the synchrotron X-ray filaments of Cas A (Uchiyama \& Aharonian 2008), which require a magnetic field amplification at the shock of the order of $\mathrm{mG}$. High-resolution observations (Gotthelf et al. 2001; Morse et al. 2004; Patnaude \& Fesen 2007; Helder \& Vink 2008) also show a reverse shock 
formed well behind the forward shock that decelerates the impinging ejecta. The parameters that characterize the reverse shock can be significantly different from the ones describing the forward shock, enhancing different dominant radiation mechanisms on each zone. For instance, inverse Compton (IC) contribution, up-scattering the large FIR photon field of Cas A itself (with an energy density of $\sim 2 \mathrm{eV} \mathrm{cm}^{3}$ and a temperature of $97 \mathrm{~K}$, Mezger et al. 1986), is more significant in a region of lower magnetic field, as otherwise it would be suppressed due to fast cooling of electrons. Hard X-ray observations (Grefenstette et al. 2015; Siegert et al. 2015), if of synchrotron origin, prove the presence of relativistic electrons with Lorentz factor $\gamma_{\mathrm{e}} \geq 100$, which can also produce gamma rays through relativistic bremsstrahlung.

We use the MAGIC telescopes to improve the accuracy of the spectral measurement at multi-TeV energies. We also derived the spectrum obtained with the LAT, selecting events with the best energy reconstruction, to extend the spectrum to lower energies and also have sufficient overlap at very high energies (VHEs). The full spectrum obtained from $\sim 60 \mathrm{MeV}$ to $\sim 10 \mathrm{TeV}$ is investigated here to determine the underlying mechanisms powering the young remnant, constraining the maximum energy of the accelerated particles and their nature.

\section{OBSERVATIONS AND DATA ANALYSIS}

\subsection{The MAGIC telescopes}

MAGIC is a system of two $17 \mathrm{~m}$ diameter Imaging Atmospheric Cherenkov Telescopes (IACTs), located at an altitude of $2200 \mathrm{~m}$ a.s.l. at the Roque de los Muchachos Observatory on the Canary Island of La Palma, Spain $\left(28^{\circ} \mathrm{N}, 18^{\circ} \mathrm{W}\right)$. The telescopes are equipped with photomultiplier tubes (PMTs) that can detect the flashes of Cherenkov light produced by extensive air showers initiated in the upper atmosphere by gamma-ray photons with energies $\gtrsim 50 \mathrm{GeV}$. In the absence of moonlight and for zenith angles less than $30^{\circ}$, MAGIC reaches an energy threshold of $\sim 50 \mathrm{GeV}$ at the trigger level and a sensitivity above $220 \mathrm{GeV}$ of $0.67 \pm 0.04$ per cent of the Crab nebula flux (C.U., Aleksić et al. 2016).

Observations were performed between 2014 December and 2016 October, for a total observation time of $158 \mathrm{~h}$ after data quality cuts. They were carried in the so-called wobble mode (Fomin et al. 1994), with a standard wobble offset of $0.4^{\circ}$. The data correspond to zenith angles between 28 and 50 degrees and most of them ( $\sim 73$ per cent) were taken during moonlight time (see Table 1), under backgroundlight levels that could be up to 12 times brighter than during dark nights. A significant part of the data ( $\sim 24$ per cent) were obtained under reduced high voltage (HV) settings: the gain of the PMTs is lowered by a factor $\sim 1.7$ to decrease the damage inflicted by the background light on the photodetectors during strong moonlight time. The main effect of moonlight in the performance of the telescopes is an increase in the energy threshold, which for zenith

Table 1. Effective observation time of the different hardware and sky brightness conditions under which Cas A samples were taken.

\begin{tabular}{lc}
\hline Observation conditions & Time [h] \\
\hline Dark and nominal HV & 42.2 \\
Moon and nominal HV & 77.7 \\
Moon and reduced HV & 38.1 \\
All configurations & 158.0 \\
\hline
\end{tabular}

angles between 30 and 45 degrees goes from $\sim 100 \mathrm{GeV}$ during dark conditions to $\sim 300 \mathrm{GeV}$ in the brightest scenario considered. As achieving a low energy threshold was not critical for this project, Moon observations provided a unique way to accumulate observation time. A detailed study of the performance of the MAGIC telescopes under moonlight is reported in MAGIC Collaboration (2017)

The data have been analysed using the standard tools used for the analysis of the MAGIC telescope data, MARS (Zanin et al. 2014) following the optimized moonlight analysis described in MAGIC Collaboration (2017). For the spectrum reconstruction, a point-like source was assumed and typical selection cuts with 90 per cent and 75 per cent $\gamma$-ray efficiency for the $\gamma$-ray/hadron separation and sky signal region radius, respectively (Aleksić et al. 2016). Three OFF regions were considered for the background estimation.

\subsection{Fermi-LAT}

The GeV emission of Cas A was revisited using 3.7 yr of LAT observations (Yuan et al. 2013). The spectrum derived is wellrepresented by a broken power law with a break of $6.9 \sigma$ significance at $\sim 1.7 \mathrm{GeV}$. To compare with the observations performed with the MAGIC telescopes, and also to update and improve the spectrum, we analysed $8.3 \mathrm{yr}$ of LAT data (up to 2016 December 6) on a $15^{\circ} \times 15^{\circ}$ region around the position of Cas $\mathrm{A}^{1}$. We selected events with energy between $60 \mathrm{MeV}$ and $500 \mathrm{GeV}$ and applied the usual filters and corrections recommended by the Fermi-LAT collaboration (removing intervals when the rocking angle of the LAT was greater than $52^{\circ}$ or when parts of the region of interest (ROI) were observed at zenith angles larger than $90^{\circ}$, as well as enabling the energy dispersion). In order to derive the energy spectrum, we applied a maximum likelihood estimation analysis in 12 independent energy bins from $60 \mathrm{MeV}$ to $500 \mathrm{GeV}$, modelling the Galactic and isotropic diffuse emission using the templates provided by the Fermi-LAT collaboration ${ }^{2}$. During the broad-band fit, all sources in the third Fermi catalogue (3FGL) within the ROI were included. A source located $\sim 3.7^{\circ}$ away from Cas A at $(1, b)=$ $\left(113.6^{\circ}, 1.1^{\circ}\right)$ was added during the fitting process to account for a significant residual excess (with TS $=45.08$ ). The spectral parameters of the background sources were fixed to those previously found, except for the sources within $5^{\circ}$ of the candidate location and the normalization of the two diffuse background components. Following the results obtained by Yuan et al. (2013), we used a smoothly broken power-law function to fit the broad-band spectrum of Cas A $\left(\mathrm{d} N / \mathrm{d} E=N_{\mathrm{o}}\left(\frac{E}{E_{o}}\right)^{\Gamma_{1}}\left(1+\left(\frac{E}{E_{\mathrm{b}}}\right)^{\left(\Gamma_{1}-\Gamma_{2}\right) / \beta}\right)^{-\beta}\right)$ with the parameter $\beta$ fixed to 1 and the energy break to $E_{\mathrm{b}}=1.7 \mathrm{GeV}$. $E_{\mathrm{o}}$ is the normalization energy, fixed to $1 \mathrm{GeV}$. The data set was reduced and analysed using Fermipy ${ }^{3}$, a set of python tools which automatize the Pass 8 analysis. We analysed the four EDISP event types separately and combined them later by means of a joint likelihood fit. The SED was obtained by fitting the source normalization factor in each energy bin independently using a power-law spectrum with a fixed spectral index of 2 . For each spectral point, we required at least a TS of 4, otherwise upper limits at the 95 per cent confidence level were computed.

\footnotetext{
${ }^{1}$ The analysis on a $30^{\circ} \times 30^{\circ}$ region yields compatible results. 2 gll_iem_v06.fits and iso_P8R2_ULTRACLEANVETO_V6_v06.txt, http://fermi.gsfc.nasa.gov/ssc/data/analysis/documentation/Cicerone ${ }^{3} \mathrm{http}: / /$ fermipy.readthedocs.org/en/latest/
} 


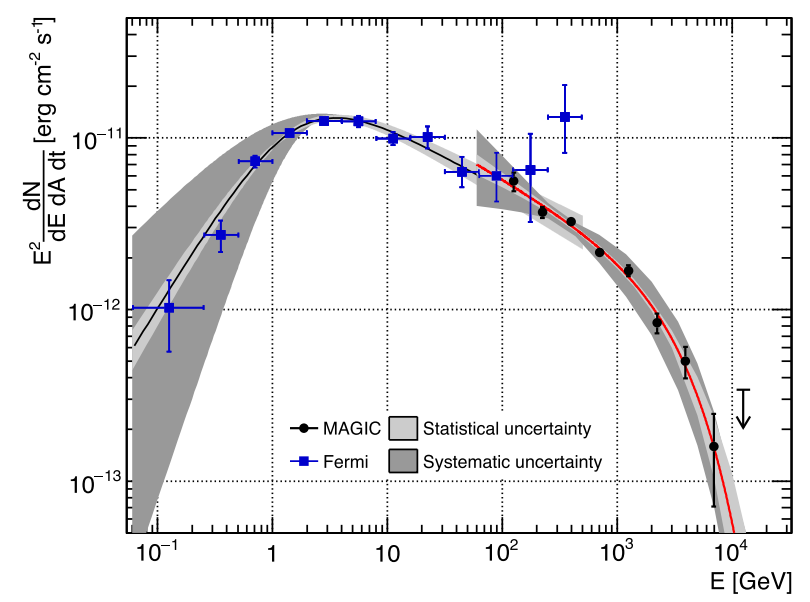

Figure 1. Spectral energy distribution measured by the MAGIC telescopes (black dots) and Fermi (blue squares). The red solid line shows the result of fitting the MAGIC spectrum with equation (1). The black solid line is the broken power-law fit applied to the Fermi spectrum.

\section{RESULTS}

Fig. 1 shows the reconstructed SED obtained with the MAGIC telescopes (black solid points). Red solid line is the curve obtained that best fits the MAGIC data assuming a power law with an exponential cut-off (EPWL):

$$
\frac{\mathrm{d} N}{\mathrm{~d} E}=N_{0}\left(\frac{E}{E_{0}}\right)^{-\Gamma} \exp \left(-\frac{E}{E_{\mathrm{c}}}\right)
$$

with a normalization constant $N_{0}=\left(1.1 \pm 0.1_{\text {stat }} \pm 0.2_{\text {sys }}\right) \times$ $10^{-11} \mathrm{TeV}^{-1} \mathrm{~cm}^{-2} \mathrm{~s}^{-1}$ at a normalization energy $E_{0}=433 \mathrm{GeV}$, a spectral index $\Gamma=2.4 \pm 0.1_{\text {stat }} \pm 0.2_{\text {sys }}$ and a cut-off energy $E_{\mathrm{c}}=3.5\left({ }_{-1.0}^{+1.6}\right)_{\text {stat }}\left({ }_{-0.9}^{+0.8}\right)_{\text {sys }} \mathrm{TeV}$. The spectral parameters of the tested models $\theta=\left\{N_{0}, \Gamma, E_{c}\right\}$ are obtained via a maximum likelihood approach. The data inputs are the numbers of recorded events (after background suppression cuts) in each bin of estimated energy $E_{\text {est }}^{i}$, both in the source direction $\left(N_{i}^{\mathrm{ON}}\right)$ and in the three OFF regions $\left(N_{i}^{\mathrm{OFF}}\right)$. An additional set of nuisance parameters $\mu_{i}$ for modelling the background are also optimized in the likelihood calculation. In each step of the maximization procedure, the expected number of gammas in a given bin of estimated energy $\left(E_{\text {est }}\right)$ is calculated by folding the gamma spectrum with the MAGIC telescopes response (energy-dependent effective area and energy migration matrix). The background nuisance parameters and the statistical uncertainties in the telescopes response are treated as explained in Rolke et al. (2005).

The probability of the EPWL fit is 0.42 . We tested the model against the null hypothesis of no cut-off, which is described with a pure power law (PWL). The probability of the PWL fit is $6 \times 10^{-4}$. A likelihood ratio test between the two tested models favours the one that includes a cut-off at $\sim 3.5 \mathrm{TeV}$ with $4.6 \sigma$ significance.

Fig. 2 compares the fit residuals for the two tested models: PWL and EPWL. The residuals are here defined as $N_{\mathrm{ON}}^{\text {obs }} / N_{\mathrm{ON}}^{\mathrm{exp}}-1$, where $N_{\mathrm{ON}}^{\mathrm{obs}}$ is the number of observed events (including background) in the $\mathrm{ON}$ region and $N_{\mathrm{ON}}^{\mathrm{exp}}$ is the number of events predicted by the fit in the same region. All the bins in estimated energy which contain events are used in the fits, but only those with $2 \sigma$ significance gamma-ray excess are shown as SED points in the upper panel of Fig. 1.

The systematic uncertainty due to an eventual mismatch on the absolute energy scale between MAGIC data and Monte Carlo (MC)


Figure 2. Relative fit residuals for the two tested models fitting the MAGIC spectrum: power law with an exponential cut-off (EPWL, upper panel) and power law (PWL, lower panel). The error bars are calculated such that they correspond to the total contribution of each estimated energy bin to the final likelihood of the fit.

simulations was constrained to be below 15 per cent in Aleksić et al. (2016). By conservatively modifying the absolute calibration of the telescopes by \pm 15 percent, and re-doing the whole analysis, we can evaluate the effect of this systematic uncertainty in the estimated source spectrum. This does not produce a simple shift of the spectrum along the energy axis, but also changes its hardness. Even in the unlikely scenario in which, through the $158 \mathrm{~h}$ of observations, the average Cherenkov light yield was overestimated by 15 per cent relative to the $\mathrm{MC}$, by applying the corresponding correction the resulting spectrum is still better fit by an EPWL at the level of $3.1 \sigma$. Also, in the unlikely scenario in which the light yield was underestimated, the EPWL is preferred over the PWL at the $6.5 \sigma$ level. The systematic uncertainties in the flux normalization and spectral index were retrieved from the publication reporting the performance of the MAGIC telescopes during moonlight (MAGIC Collaboration 2017). The systematic errors in the cut-off energy were estimated from the values of $E_{\mathrm{c}}$ obtained when modifying the absolute light scale by \pm 15 per cent.

For the Fermi-LAT analysis, a broken power-law function with normalization $N_{\mathrm{o}}=(8.0 \pm 0.4) \times 10^{-12} \mathrm{MeV}^{-1} \mathrm{~cm}^{-2} \mathrm{~s}^{-1}$ and indices $\Gamma_{1}=0.90 \pm 0.08$ and $\Gamma_{2}=2.37 \pm 0.04$ is obtained and shown in Fig. 1 as blue solid squares. The light grey shaded area shows the statistical errors of the obtained broken power-law fit whereas the dark one marks the uncertainty coming from the imperfectness in the Galactic diffuse emission modelling, dominating the Cas A flux uncertainties at low energies. The latter was obtained by modifying the galactic diffuse flux by \pm 6 per cent. Note that the systematic error due to the diffuse background is greatly reduced above $300 \mathrm{MeV}$.

\section{DISCUSSION}

MAGIC observations of the youngest $\mathrm{GeV}$ - and TeV-bright known SNR have allowed us to obtain the most precise spectrum of Cas A to date, extending previous results obtained with Cherenkov instruments up to $\sim 10 \mathrm{TeV}$. In the MAGIC energy range, the spectrum is best fitted with a power law with an exponential cut-off function with an index of $\sim 2.4$ and an energy cut-off at $E_{\mathrm{c}} \sim 3.5 \mathrm{TeV}$. These findings provide a crucial insight into the acceleration processes in one of the most prominent non-thermal objects in our Galaxy.

We also analysed more than $8 \mathrm{yr}$ of LAT data and obtained a spectrum that confirms the one by Yuan et al. (2013). Below 




Figure 3. Multiwavelength SED of Cas A. The different lines show the result of fitting the measured energy fluxes using naima and assuming a leptonic or a hadronic origin of the $\mathrm{GeV}$ and $\mathrm{TeV}$ emission.

$\sim 1 \mathrm{GeV}$, Cas A shows a hard spectrum with an index of $\sim 0.9$. Above a few $\mathrm{GeV}$, the spectrum measured with Fermi-LAT falls quickly with a photon index of $\sim 2.37$, which is compatible within errors with the one measured using the MAGIC telescopes.

To investigate the underlying population of particles, we have used the radiative code and Markov Chain Monte Carlo fitting routines of naima ${ }^{4}$ (Zabalza 2015), deriving the present-age particle distribution. The code uses the parametrization of neutral pion decay by Kafexhiu et al. (2014), the parametrization of synchrotron radiation by Aharonian, Kelner \& Prosekin (2010) and the analytical approximations to IC up-scattering of black body radiation and non-thermal bremsstrahlung developed by Khangulyan, Aharonian \& Kelner (2014) and Baring et al. (1999), respectively.

We first considered the possibility that the gamma-ray emission was originated by an electron population, described with a power law with an exponential cut-off function, producing Bremsstrahlung and IC radiation in the gamma-ray range and synchrotron radiation at lower energies. The photon fields that contribute to the inverse Compton component are the ubiquitous $2.7 \mathrm{~K}$ cosmic microwave background (CMB) and the large far-infrared (FIR) field measured in Cas A, with a value of $\sim 2 \mathrm{eVcm}^{-3}$ at $100 \mathrm{keV}$. Fixing the photon field to this value, we can obtain the highest possible density of electrons allowed by the VHE flux. Then we can constrain the maximum magnetic field for which the synchrotron radiation produced by the derived population does not exceed the radio and X-ray

${ }^{4}$ https://github.com/zblz/naima measurements ${ }^{5}$. The multiwavelength SED is shown in Fig. 3, with the radio emission displayed in purple dots (Lastochkin et al. 1963; Medd \& Ramana 1965; Allen \& Barrett 1967; Parker 1968; Braude et al. 1969; Hales et al. 1995; Planck Collaboration XXVIII 2014), soft SUZAKU X-rays are marked in red (Maeda et al. 2009) and hard INTEGRAL X-rays in blue (Wang \& Li 2016). In the gammaray regime, the LAT points are shown in cyan and the MAGIC ones in green. The MAGIC points can be described by an electron population with an amplitude at $1 \mathrm{TeV}$ of $2 \cdot 10^{34} \mathrm{eV}^{-1}$, a spectral index of 2.4 and cut-off energy at $8 \mathrm{TeV}$ up-scattering the FIR (brown dashdot line) and the CMB photons (green dashed line). The comparison with the low energy part of the SED constrains the magnetic field to $B \lesssim 180 \mu \mathrm{G}$. The resulting emission from the leptonic model is shown in Fig. 3. A relatively low magnetic field and a large photon field could be fulfilled in a reverse shock evolving in a thin and clumpy ejecta medium which provides a moderate amplification of the magnetic field and large photon fields in the clumps that are observed as optical knots. The same population of electrons would unavoidably produce Bremsstrahlung radiation below a few $\mathrm{GeV}$ (see green dotted line in Fig. $3^{6}$ ). The emission observed with FermiLAT at the lowest energies constrains the density to $n \sim 1 \mathrm{~cm}^{-3}$,

\footnotetext{
${ }^{5}$ This constraint is due to the fact that, as reported in Section 1, several emission regions, likely associated to different particle populations, were identified at those wavelengths.

${ }^{6}$ Note that the structure in the spectral shape around $2 \mathrm{MeV}$ is due to the transition between the two asymptotic regimes described in Baring et al. (1999), used in the naima code.
} 
still compatible with the smooth ejecta density (Micelotta, Dwek \& Slavin 2016). The model is generally compatible with the Xray points and with the MAGIC spectrum above a few $\mathrm{TeV}$; it is consistent with the radio measurements, but fails to reproduce the $\gamma$-ray spectrum between $1 \mathrm{GeV}$ and $1 \mathrm{TeV}$, being a factor $2-3$ below the measured LAT spectrum. In addition, to accommodate a magnetic field of the order of $\sim 1 \mathrm{mG}$, as reported in Uchiyama \& Aharonian (2008), the amplitude of the electron spectrum would need to be decreased at least by a factor 100 , rendering a negligible IC contribution at the highest energies.

Indeed the $\mathrm{GeV}-\mathrm{TeV}$ emission of Cas A is usually attributed to accelerated protons. Assuming a population of CRs characterized with a power-law function with an exponential cut-off to fit the gamma-ray data from $60 \mathrm{MeV}$ to $15 \mathrm{TeV}$ and a target density of $10 \mathrm{~cm}^{-3}$ (Laming \& Hwang 2003), the proton spectrum is best fitted with a hard index of 2.21 and an exponential cut-off energy of $12 \mathrm{TeV}$, which implies a modest acceleration of CRs to VHE is well below the energy needed to explain the CR knee. The proton energy above $1 \mathrm{TeV}$ is $5.1 \cdot 10^{48} \mathrm{erg}$, which is only $\sim 0.2$ percent of the estimated explosion kinetic energy of $E_{\mathrm{sn}}=2 \cdot 10^{51} \mathrm{erg}$ (Laming $\&$ Hwang 2003). The total energy stored in protons above $100 \mathrm{MeV}$ amounts to $9.9 \cdot 10^{49} \mathrm{erg}$.

The flat spectral index is in agreement with the standard theory of diffuse shock acceleration, but the low cut-off energy implies that Cas A is extremely inefficient in the acceleration of CRs at the present moment. The characteristic maximum energy of these accelerated protons can be expressed, for standard parallel shock acceleration efficiency (see e.g. Lagage \& Cesarsky 1983), as:

$E_{\mathrm{c}}^{\mathrm{p}} \simeq 450\left(\frac{B}{1 \mathrm{mG}}\right)\left(\frac{t_{0}}{100 \mathrm{yr}}\right)\left(\frac{u_{\mathrm{s}}}{3000 \mathrm{kms}}\right)^{2} \eta^{-1} \mathrm{TeV}$,

where $u_{\mathrm{s}} \sim 10^{3} \mathrm{~km} \mathrm{~s}^{-1}$ is the speed of the forward shock, $t_{0} \sim 330 \mathrm{yr}$ is the age of Cas $\mathrm{A}$ and $\eta \geq 1$ is the acceleration efficiency (the ratio of the mean free path of a particle to its gyroradius), which is $\sim 1$ in the Bohm diffusion regime. Even assuming a magnetic field as low as a few tens of $\mu \mathrm{G}$, a poor acceleration efficiency $\eta \gg 10$ has to be invoked to accommodate the low cut-off energy found. Alternatively, Cas A may also be located in a very diffusive region of the Galaxy, resulting in a very fast escape of protons of $\mathrm{TeV}$ and higher energies.

\section{CONCLUSIONS}

We report for the first time in VHE, observational evidence for the presence of a cut-off in the VHE spectrum of Cas A. The spectrum measured with the MAGIC telescopes can be described with an EPWL with a cut-off at $\sim 3.5 \mathrm{TeV}$, which is preferred over a PWL scenario with $4.6 \sigma$ significance. This result implies that even if all the $\mathrm{TeV}$ emission was of hadronic origin, Cas A could not be a PeVatron at its present age.

Several emission regions must be active to explain the radio, $\mathrm{X}$-ray, $\mathrm{GeV}$ and $\mathrm{TeV}$ emission of Cas A. A purely leptonic model cannot explain the $\mathrm{GeV}-\mathrm{TeV}$ spectral shape derived using LAT and MAGIC data, as previously suggested based on observations at lower energies (Atoyan et al. 2000a,b; Saha et al. 2014; Zirakashvili et al. 2014). A leptonic population is undoubtedly necessary to explain the emission at radio and X-ray energies. Indeed, the bright steep-spectrum radio knots and the bright radio ring demand an average magnetic field of $\sim 1 \mathrm{mG}$ (Vink \& Laming 2003), whereas the faint plateau surrounding Cas A, seen in Chandra continuum images, is consistent with a lower magnetic field, which might contribute to the observed emission above $1 \mathrm{TeV}$.
However, the bulk of the HE and VHE $\gamma$-rays must be of hadronic origin. Cas A is most likely accelerating CRs, although to a rather low energy of a few $\mathrm{TeV}$. Even if some leptonic contribution at VHE produced by IC cannot be excluded, this would not affect our conclusion that acceleration in Cas A falls short of the energies of the knee of the CR spectrum.

A detailed study of the cut-off shape is crucial to understand the reason behind this low acceleration efficiency, displaying different characteristics if due to escape of CRs, to the maximum energy of the accelerated CRs, or some other mechanism. Observations with the future Cherenkov Telescope Array (CTA, Actis et al. 2011), with a superior angular resolution and sensitivity, will allow a detailed spectroscopic investigation on the cut-off regime, providing new insights into the acceleration processes in Cas A.

\section{ACKNOWLEDGEMENTS}

We would like to thank the Instituto de Astrofísica de $\mathrm{Ca}$ narias for the excellent working conditions at the Observatorio del Roque de los Muchachos in La Palma. The financial support of the German BMBF and MPG, the Italian INFN and INAF, the Swiss National Fund (SNF), the ERDF under the Spanish MINECO (FPA2015-69818-P, FPA2012-36668, FPA2015-68378-P, FPA2015-69210-C6-2-R, FPA2015-69210-C64-R, FPA2015-69210-C6-6-R, AYA2015-71042-P, AYA201676012-C3-1-P, ESP2015-71662-C2-2-P and CSD2009-00064) and the Japanese JSPS and MEXT is gratefully acknowledged. This work was also supported by the Spanish Centro de Excelencia 'Severo Ochoa' SEV-2012-0234 and SEV-2015-0548, Unidad de Excelencia 'María de Maeztu' MDM-2014-0369, by the Croatian Science Foundation (HrZZ) Project 09/176, the University of Rijeka Project 13.12.1.3.02, by the DFG Collaborative Research Centers SFB823/C4 and SFB876/C3 and by the Polish MNiSzW grant 745/N-HESS-MAGIC/2010/0.

\section{REFERENCES}

Abdo A. A. et al., 2010, ApJ, 710, L92

Ackermann M. et al., 2013, Science, 339, 807

Actis M. et al., 2011, Exp. Astron., 32, 193

Aharonian F., 2013, Astropart. Phys., 43, 71

Aharonian F. et al., 2001, A\&A, 370, 112

Aharonian F. A., Kelner S. R., Prosekin A. Y., 2010, Phys. Rev., 82, 043002 Albert J. et al., 2007, A\&A, 474, 937

Aleksić J. et al., 2016, Astropart. Phys., 72, 76

Allen R. J., Barrett A. H., 1967, ApJ, 149, 1

Anderson M. C., Rudnick L., 1995, ApJ, 441, 307

Anderson M., Rudnick L., Leppik P., Perley R., Braun R., 1991, ApJ, 373, 146

Atoyan A. M., Aharonian F. A., Tuffs R. J., Völk H. J., 2000a, A\&A, 355, 211

Atoyan A. M., Tuffs R. J., Aharonian F. A., Völk H. J., 2000b, A\&A, 354, 915

Axford W. I., Leer E., Skadron G., 1977, Proc. 15th Int. Cosm. Ray Conf. (ICRC), Vol. 11, The Acceleration of Cosmic Rays by Shock Waves. Plovdiv, Bulgaria, Bulgarian p.132

Baring M. G., Ellison D. C., Reynolds S. P., Grenier I. A., Goret P., 1999, ApJ, 513, 311

Bell A. R., 1978, MNRAS, 182, 147

Bell A. R., 1978, MNRAS, 182, 443

Bell A. R., 2004, MNRAS, 353, 550

Bell A. R., 2013, Astropart. Phys., 43, 56

Bell A. R., Schure K. M., Reville B., Giacinti G., 2013, MNRAS, 431, 415

Berezhko E. G., Pühlhofer G., Völk H. J., 2003, A\&A, 400, 971 
Blandford R. D., Ostriker J. P., 1978, ApJ, 221, L29

Braude S. Y., Lebedeva O. M., Megn A. V., Ryabov B. P., Zhouck I. N., 1969, MNRAS, 143, 289

Cowsik R., Sarkar S., 1980, MNRAS, 191, 855

DeLaney T., Rudnick L., 2003, ApJ, 589, 818

Fomin V. P., Stepanian A. A., Lamb R. C., Lewis D. A., Punch M., Weekes T. C., 1994, Astropart. Phys., 2, 137

Gaisser T. K., 1991, Cosmic Rays and Particle Physics, Cambridge Univ. Press, Cambridge

Ginzburg V. L., Syrovatskii S. I., 1964, The Origin of Cosmic Rays, Macmillan, New York

Gotthelf E. V., Koralesky B., Rudnick L., Jones T. W., Hwang U., Petre R., 2001, ApJ, 552, L39

Grefenstette B. W. et al., 2015, ApJ, 802, 15

Hales S. E. G., Waldram E. M., Rees N., Warner P. J., 1995, MNRAS, 274 , 447

Helder E. A., Vink J., 2008, ApJ, 686, 1094

Holder J., 2017, in AIP Conf. Proc., 1792, 020013

Kafexhiu E., Aharonian F., Taylor A. M., Vila G. S., 2014, Phys. Rev., 90, 123014

Khangulyan D., Aharonian F. A., Kelner S. R., 2014, ApJ, 783, 100

Krymskii G. F., 1977, Akademiia Nauk SSSR Dokl., 234, 1306

Kumar S. for the VERITAS Collaboration, 2015, Proc. 34th Int. Cosm. Ray Conf. The Hague, The Netherlands

Lagage P. O., Cesarsky C. J., 1983, A\&A, 125, 249

Laming J. M., Hwang U., 2003, ApJ, 597, 347

Lastochkin V. P., Porfriev V. A., Stankevich K. S., Troitsky V. S., Kholodilov N. N., Tseitlin N. M., 1963, Radiophysica (U.S.S.R.), 6, 629

Maeda Y. et al., 2009, PASJ, 61, 1217

MAGIC Collaboration: Ahnen M. L. et al., 2017, Astropart. Phys., 94, 29

Medd W. J., Ramana K. V. V., 1965, ApJ, 142, 383

Mezger P. G., Tuffs R. J., Chini R., Kreysa E., Gemuend H.-P., 1986, A\&A, 167,145

Micelotta E. R., Dwek E., Slavin J. D., 2016, A\&A, 590, A65

Morse J. A., Fesen R. A., Chevalier R. A., Borkowski K. J., Gerardy C. L., Lawrence S. S., van den Bergh S., 2004, ApJ, 614, 727

O’C. Drury L., 2014, Invited talk at CRISM2014 Conf. Montpellier, France Parker E. A., 1968, MNRAS, 138, 407

Patnaude D. J., Fesen R. A., 2007, AJ, 133, 147

Planck Collaboration XXVIII, 2014, A\&A, 571, A28

Reed J. E., Hester J. J., Fabian A. C., Winkler P. F., 1995, ApJ, 440, 706

Rolke W. A., López A. M., Conrad J., 2005, Nucl. Instrum. Methods Phys. Res., 551, 493

Saha L., Ergin T., Majumdar P., Bozkurt M., Ercan E. N., 2014, A\&A, 563, A88

Siegert T., Diehl R., Krause M. G. H., Greiner J., 2015, A\&A, 579, A124

Uchiyama Y., Aharonian F. A., 2008, ApJ, 677, L105

Vink J., Laming J. M., 2003, ApJ, 584, 758

Wang W., Li Z., 2016, ApJ, 825, 102

Yuan Y., Funk S., Johannesson G., Lande J., Tibaldo L., Uchiyama Y., 2013, ApJ, 779, 117
Zabalza V., 2015, in Proc. 34th Int. Cosm. Ray Conf. The Hague, The Netherlands p.922

Zanin R., Carmona E., Sitarek J., Colin P., Frantzen K., 2014, Braz. J. Phys., 44,415

Zirakashvili V. N., Aharonian F. A., Yang R., Oña-Wilhelmi E., Tuffs R. J., 2014, ApJ, 785, 130

${ }^{1}$ ETH Zurich, CH-8093 Zurich, Switzerland

${ }^{2}$ Università di Udine and INFN Trieste, I-33100 Udine, Italy

${ }^{3}$ INAF-National Institute for Astrophysics, viale del Parco Mellini, 84, I-00136 Rome, Italy

${ }^{4}$ Università di Padova and INFN, I-35131 Padova, Italy

${ }^{5}$ Croatian MAGIC Consortium, Rudjer Boskovic Institute, University of Rijeka, University of Split-FESB, University of Zagreb-FER, University of Osijek, Croatia

${ }^{6}$ Saha Institute of Nuclear Physics, 1/AF Bidhannagar, Salt Lake, Sector-1, Kolkata 700064, India

${ }^{7}$ Max-Planck-Institut für Physik, D-80805 München, Germany

${ }^{8}$ Universidad Complutense, E-28040 Madrid, Spain

${ }^{9}$ Dpto. Astrofísica, Inst. de Astrofísica de Canarias, E-38200 and Universidad de La Laguna, E-38206 La Laguna, Tenerife, Spain

${ }^{10}$ University of Łódź, PL-90236 Lodz, Poland

${ }^{11}$ Deutsches Elektronen-Synchrotron (DESY), D-15738 Zeuthen, Germany

${ }^{12}$ Institut für Physik Newtonstr. 15, Humboldt University of Berlin, D-12489 Berlin, Germany

${ }^{13}$ Institut de Fisica d'Altes Energies (IFAE), The Barcelona Institute of Science and Technology, Campus UAB, E-08193 Bellaterra (Barcelona), Spain

${ }^{14}$ Università di Siena and INFN Pisa, I-53100 Siena, Italy

${ }^{15}$ Institute for Space Sciences (CSIC/IEEC), E-08193 Barcelona, Spain

${ }^{16}$ Technische Universität Dortmund, D-44221 Dortmund, Germany

${ }^{17}$ Universität Würzburg, D-97074 Würzburg, Germany

${ }^{18}$ Finnish MAGIC Consortium: Tuorla Observatory, University of Turku and Astronomy Division, University of Oulu, Finnish Centre for Astronomy with ESO (FINCA), Finland

${ }^{19}$ Unitat de Física de les Radiacions, Departament de Física and CERESIEEC, Universitat Autònoma de Barcelona, E-08193 Bellaterra, Spain

${ }^{20}$ Universitat de Barcelona, ICC, IEEC-UB, E-08028 Barcelona, Spain

${ }^{21}$ Japanese MAGIC Consortium, ICRR, The University of Tokyo, Department of Physics, Kyoto University, Tokai University, The University of Tokushima, Japan

${ }^{22}$ Institute for Nuclear Research and Nuclear Energy, BG-1784 Sofia, Bulgaria

${ }^{23}$ Università di Pisa and INFN Pisa, I-56126 Pisa, Italy

This paper has been typeset from a $\mathrm{TE}_{\mathrm{E}} \mathrm{X} / \mathrm{A} \mathrm{T} \mathrm{E}$ file prepared by the author. 